\title{
Diffractive charged meson pair production
}

\author{
B. Lehmann-Dronke ${ }^{\text {a }}$, M. Maul ${ }^{\text {b }}$, S. Schaefer ${ }^{\text {a }}$, E. Stein ${ }^{a}$, \\ A. Schäfer ${ }^{\text {a }}$ \\ ${ }^{a}$ Institut für Theoretische Physik, Universität Regensburg, D-93040 Regensburg, \\ Germany \\ ${ }^{\mathrm{b}}$ Nordic Institute for Theoretical Physics, Blegdamsvej 17, DK-2100 Copenhagen, \\ Denmark
}

\begin{abstract}
We investigate the possibility to measure the nonforward gluon distribution function by means of diffractively produced $\pi^{+} \pi^{-}$and $K^{+} K^{-}$pairs in polarized lepton nucleon scattering. The resulting cross sections are small and are dominated by the gluonic contribution. We find relatively large spin asymmetries, both for $\pi^{+} \pi^{-}$and for $K^{+} K^{-}$pairs.
\end{abstract}

PACS. 12.38.-t, 13.60.-r, 13.60.Le

\section{Introduction}

QCD parton distribution functions are specified by matrix-elements of nonlocal quark and gluon twist-2 operators separated by a light like distance and taken inside a forward nucleon matrix element. Although these forward matrix elements were generalized long time ago to nonforward parton distribution functions [1], where nonlocal QCD operators are sandwiched between states of unequal momenta, they became only recently the object of intense studies [24]. This interest was driven by the hope that the measurement of nonforward parton distributions (NFPDs) and subsequent extrapolation to the forward limit might shed more light on the spin and angular momentum composition of the nucleon [5].

Contrary to ordinary parton distribution functions, nonforward parton distribution functions are probed when the nucleon recoils elastically. Thus NFPDs provide a natural interpolation between ordinary parton distribution functions and elastic form factors. It was shown that NFPDs are in principle measurable in deeply virtual Compton scattering or through exclusive production of light vector mesons. 


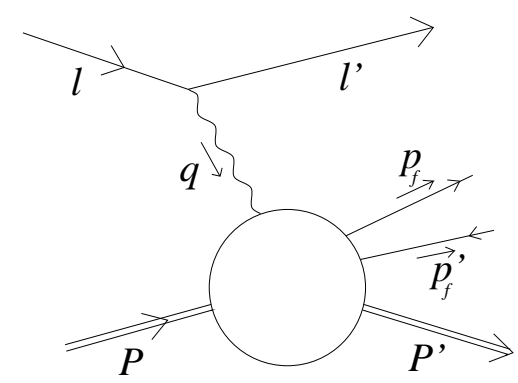

Fig. 1. The process of the quark antiquark pair production to lowest order QED.

In this paper we shall consider the diffractive leptoproduction of quark antiquark pairs in a kinematics where the produced quark pair does not form a single meson but fragments into a $\pi^{+} \pi^{-}$or $K^{+} K^{-}$pair with comparatively large $p_{T}$ (Fig. 1). We will consider the case of longitudinal polarized electron beam and proton target. This process is quite similar to diffractive leptoproduction of dijets, analyzed in [6,7], which was proposed to allow for a measurement of the polarized glue in the nucleon. We notice that at medium energies like in the HERMES experiment dijets cannot be observed. In this experiment one can only look for single hadrons representing the jets. The idea to access the polarized forward gluon distribution function of the nucleon via the measurement of a double hadronic high momentum final state has been proposed in [8].

We treat the fragmentation process of the two produced quarks into the final hadrons using the LUND string fragmentation model $[9,10]$. To be precise we use this model to extrapolate from dijet production, where it is known to be valid, to the case where the two jets are represented by e.g. $\pi^{+}$and $\pi^{-}$. While we do not expect the LUND model to give precise results in this few particle process it should allow for an order of magnitude estimate.

In our calculation we will assume that two pion (kaon) leptoproduction can be factorized into three parts. We have two soft parts: one describing the fragmentation of the two partons, the other the production of two partons from the elastic scattering off the nucleon. The second soft part will be given by a nucleon matrix element of a light cone operator parameterized by NFPDs. The hard part connects the elastic scattering process and subsequent fragmentation.

Technically, the process we consider is closely related to the exclusive production of a light vector meson. In such a case the proof of factorization has been given in [11]. Recall that the proof only holds for longitudinal photons. Meson production by a transversely polarized photon is plagued by endpoint singularities as noted in [12]. Thus a general factorization theorem does not exist in this case. Further the factorization theorem was proven only on the level of twist-2. However, it was noted that the proof in [11] can be extended to two-pion production if these are produced close to threshold, i.e. if the mass 


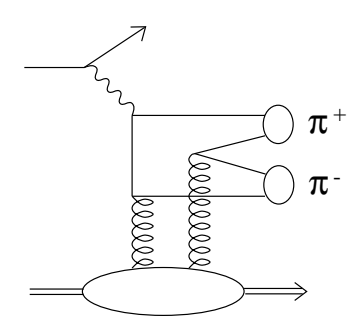

Fig. 2. diagram for the production of a pion pair by the fragmentation of four quarks.

of the produced final state is much smaller than the virtuality of the probing photon [13]. In such a kinematics it is useful to describe the pion pair in terms of a two-pion twist-2 distribution amplitude [14]. The main difference to our calculation then is the treatment of the produced meson in terms of a light cone twist- 2 meson wave function rather than in terms of the fragmentation model. In our calculation we do not restrict ourselves to the kinematic region $M^{2}\left(h^{+}+h^{-}\right)<Q^{2}$ because we have to avoid too small invariant masses in the final quark state to justify the application of the LUND fragmentation model and for correspondingly large $Q^{2}$ the counting rates are too small for an experiment like HERMES. However, in our kinematics a hard scale is given by $\mu^{2}=p_{T}^{2}\left(1+\frac{Q^{2}}{M^{2}\left(h^{+}+h^{-}\right)}\right)$with $p_{T}$ being the transverse momentum of the produced quark in the center of mass system of the incoming nucleon and the virtual photon [15]. The hardness of this scale ensures the applicability of perturbation theory. The calculation therefore can be considered as a parton model description combined with the LUND fragmentation model.

We would like to stress that the process we are considering is not the only one leading to two charged pions in the final state. An exclusively produced $\varrho$-Meson for instance will immediately decay into a pion-pair. However, the cuts in our numerical analysis make sure that we only count pions produced away from the $\varrho$-resonance. A pion pair can also be produced through the exclusive production of four quarks, each pair forming a meson! $\square$. A leading order diagram for this process is shown in Fig. 2. However, one may expect that such diagrams are kinematically suppressed as can be seen best in the center of mass frame of the incoming nucleon and the virtual photon: two of the four produced quarks are obtained by the decay of a gluon taken out of the nucleon and therefore will carry a fraction of the incoming proton momentum while the other quark antiquark pair carries the momentum of the virtual photon. Without at least one additional internal gluon line, which would lead to a suppression in $\alpha_{S}$, it is not possible to rearrange the momenta such that two light mesons are formed. Also we did not consider the process of two gluon production and their fragmentation into a meson pair. However, it turns out that in the considered kinematical range contributions from gluon nonforward parton distributions dominate compared to quark nonforward distributions whereas only the latter lead to two gluon production in the leading order.

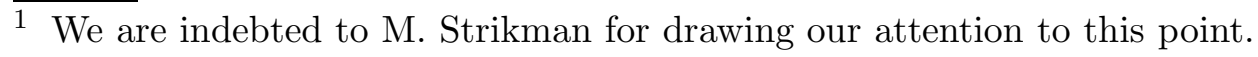




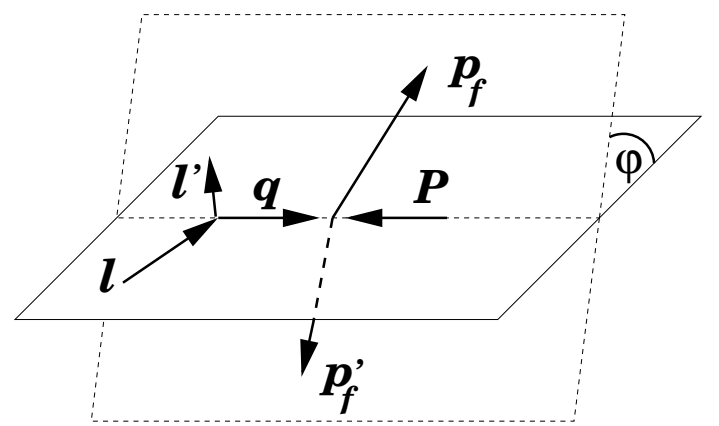

Fig. 3. Kinematics in the boson-nucleon center of mass frame.

The paper is organized as follows: First, we define the general kinematics, give the formula for the partonic cross section and derive the amplitudes for exclusive $q \bar{q}$ production. We then discuss the models used for the polarized and unpolarized NFPDs. Subsequently, we discuss the fragmentation process of the two final partons into the meson pair using PYTHIA/JETSET [10].

\section{Cross Section for Diffractive $q \bar{q}$ Production}

As already mentioned we assume that meson pair production factorizes into hard quark pair production and a fragmentation part. We treat the fragmentation process on the level of transition probabilities using the program JETSET. Therefore we have to calculate analytically only the cross section for the process

$$
e(l)+N(P) \rightarrow e\left(l^{\prime}\right)+q\left(p_{f}\right)+\bar{q}\left(p_{f}^{\prime}\right)+N^{\prime}\left(P^{\prime}\right)
$$

The corresponding diagram to lowest order QED is shown in Fig. 1. For the definition of the particle momenta see also that figure. We work in the center of mass frame of the exchanged photon and the initial nucleon and neglect nucleon and lepton masses. Furthermore we take the momentum transfer to the nucleon to be proportional to the initial nucleon momentum $P_{\mu}-P_{\mu}^{\prime}=\zeta P_{\mu}$. We have chosen the momenta $\vec{q}$ and $\vec{P}$ to define the $z$ - and $-z$-direction respectively as explained in [16]. See also Fig. 3.

The masses of the final quarks are neglected $p_{f}^{2}=p_{f}^{\prime 2}=0$. Recall that for exclusive production of only one particle the on-shell condition leads to the constraint that the skewedness parameter of the NFPD $\zeta$ equals the Bjorken variable $x_{\mathrm{Bj}}=-q^{2} / 2 \nu, \nu=P \cdot q[17]$. This is clearly not the case for two produced particles. Therefore it is natural to introduce the variable $x_{p}=x_{\mathrm{Bj}} / \zeta$, $0 \leq x_{p} \leq 1$ which describes the invariant mass of the final $q \bar{q}$ state. Using 
furthermore the variables

$$
Q^{2}=-q^{2}, y=\frac{P \cdot q}{P \cdot l}, z_{f}=\frac{P \cdot p_{f}}{P \cdot q}, t=\left(P-P^{\prime}\right)^{2}
$$

and $\varphi$ defined as the difference between the azimuthal angles of $l$ and $p_{f}$ (see Fig. 3) we get for the differential cross section

$$
\frac{\mathrm{d} \sigma}{\mathrm{d} x_{\mathrm{Bj}} \mathrm{d} y \mathrm{~d} \zeta \mathrm{d} z_{f} \mathrm{~d} t \mathrm{~d} \varphi}=\frac{\alpha_{e m}^{2}}{Q^{4}}\left(\frac{1}{4 \pi}\right)^{4} y L^{\mu \nu} H_{\mu \nu}
$$

where $L^{\mu \nu}$ is the usual leptonic tensor given by

$$
\begin{aligned}
L^{\mu \nu} & =\frac{1}{2} \sum_{\lambda^{\prime}} \bar{u}_{\lambda}(l) \gamma^{\mu} u_{\lambda^{\prime}}\left(l^{\prime}\right) \bar{u}_{\lambda^{\prime}}\left(l^{\prime}\right) \gamma^{\nu} u_{\lambda}(l) \\
& =2 l^{\mu} l^{\nu}-l^{\mu} q^{\nu}-l^{\nu} q^{\mu}-\frac{1}{2} Q^{2} g^{\mu \nu}-\lambda \mathrm{i} \varepsilon^{\mu \nu \varrho \sigma} l_{\varrho} q_{\sigma} .
\end{aligned}
$$

Summing over the spins of the final nucleon we define analogously the hadronic tensor as $\sum_{S^{\prime}} H_{\mu \nu}=T_{\mu}^{*} T_{\nu}$ with

$$
\begin{aligned}
& (2 \pi)^{4} \delta\left(p_{f}+p_{f}^{\prime}-q-\zeta P\right) T_{\mu} \\
& =\int \mathrm{d}^{4} x \mathrm{e}^{-\mathrm{i} q \cdot x}\left\langle q\left(p_{f}\right) \bar{q}\left(p_{f}^{\prime}\right) N\left(P^{\prime}\right)\left|j_{\mu}(x)\right| N(P)\right\rangle .
\end{aligned}
$$

$j_{\mu}(x)$ denotes the vector current that couples to the virtual photon. In second order perturbation theory we may write

$$
\begin{aligned}
& (2 \pi)^{4} \delta\left(p_{f}+p_{f}^{\prime}-q-\zeta P\right) T_{\mu}=\frac{\mathrm{i}^{2} g^{2}}{2 !} \int \mathrm{d}^{4} x_{1} \int \mathrm{d}^{4} x_{2} \int \mathrm{d}^{4} x_{3} \mathrm{e}^{-\mathrm{i} q \cdot x_{1}} \\
& \times\left\langle q\left(p_{f}\right) \bar{q}\left(p_{f}^{\prime}\right) N\left(P^{\prime}\right)\left|T\left\{j_{\mu}\left(x_{1}\right) j_{\alpha}^{a}\left(x_{2}\right) j_{\beta}^{b}\left(x_{3}\right) A^{a \alpha}\left(x_{2}\right) A^{b \beta}\left(x_{3}\right)\right\}\right| N(P)\right\rangle
\end{aligned}
$$

with the color current $j_{\alpha}^{a}$ and the gluon field $A^{a \alpha}$. We factorize quark and gluon fields in hard propagators and non perturbative matrix elements respectively using the parameterization of nonforward matrix elements of bilocal operators in terms of nonforward parton distributions [18]. We project onto the twist-2 contributions by introducing a light-like vector $q_{1}^{2}=\left(q+x_{\mathrm{Bj}} P\right)^{2}=0$ so that we have for the quark matrix element

$$
\begin{aligned}
& \left\langle N\left(P^{\prime}\right)\left|\bar{\psi}_{f}\left(x_{2}\right) \phi_{1} \psi_{f}\left(x_{1}\right)\right| N(P)\right\rangle=\bar{U}\left(P^{\prime}\right) \phi_{1} U(P) \\
& \times \int \mathrm{d} X\left[\mathcal{F}_{\zeta}^{f}(X) \mathrm{e}^{-\mathrm{i} X P \cdot x_{1}+\mathrm{i}(X-\zeta) P \cdot x_{2}}-\mathcal{F}_{\zeta}^{\bar{f}}(X) \mathrm{e}^{-\mathrm{i} X P \cdot x_{2}+\mathrm{i}(X-\zeta) P \cdot x_{1}}\right] \\
& +\mathcal{K} \text {-terms }
\end{aligned}
$$




$$
\begin{aligned}
& \left\langle N\left(P^{\prime}\right)\left|\bar{\psi}_{f}\left(x_{2}\right) \gamma_{5} \phi_{1} \psi_{f}\left(x_{1}\right)\right| N(P)\right\rangle=\bar{U}\left(P^{\prime}\right) \gamma_{5} \phi_{1} U(P) \\
& \times \int \mathrm{d} X\left[\Delta \mathcal{F}_{\zeta}^{f}(X) \mathrm{e}^{-\mathrm{i} X P \cdot x_{1}+\mathrm{i}(X-\zeta) P \cdot x_{2}}+\Delta \mathcal{F}_{\zeta}^{\bar{f}}(X) \mathrm{e}^{-\mathrm{i} X P \cdot x_{2}+\mathrm{i}(X-\zeta) P \cdot x_{1}}\right] \\
& +\mathcal{K} \text {-terms }
\end{aligned}
$$

and for the gluon fields

$$
\begin{aligned}
& \left\langle N\left(P^{\prime}\right)\left|A_{\varrho}^{a}\left(x_{2}\right) A_{\sigma}^{a}\left(x_{1}\right)\right| N(P)\right\rangle \\
& =\frac{\bar{U}\left(P^{\prime}\right) \phi_{1} U(P)}{2 P q_{1}}\left(-g_{\varrho \sigma}+\frac{P_{\varrho} q_{1 \sigma}+P_{\sigma} q_{1}}{P q_{1}}\right) \\
& \times \int \frac{\mathrm{d} X}{2} \frac{\mathcal{G}_{\zeta}(X)}{(X-\zeta+\mathrm{i} \varepsilon)(X-\mathrm{i} \varepsilon)}\left[\mathrm{e}^{-\mathrm{i} X P \cdot x_{1}+\mathrm{i}(X-\zeta) P \cdot x_{2}}+\mathrm{e}^{-\mathrm{i} X P \cdot x_{2}+\mathrm{i}(X-\zeta) P \cdot x_{1}}\right] \\
& +\frac{\bar{U}\left(P^{\prime}\right) \gamma_{5} q_{1} U(P)}{2 P q_{1}} \mathrm{i} \varepsilon_{\varrho \sigma \lambda \eta} \frac{P^{\lambda} q_{1}^{\eta}}{P \cdot q_{1}} \\
& \times \int \frac{\mathrm{d} X}{2} \frac{\Delta \mathcal{G}_{\zeta}(X)}{(X-\zeta+\mathrm{i} \varepsilon)(X-\mathrm{i} \varepsilon)}\left[\mathrm{e}^{-\mathrm{i} X P \cdot x_{1}+\mathrm{i}(X-\zeta) P \cdot x_{2}}-\mathrm{e}^{-\mathrm{i} X P \cdot x_{2}+\mathrm{i}(X-\zeta) P \cdot x_{1}}\right] \\
& +\mathcal{K}-\text { terms } .
\end{aligned}
$$

We have not written the gauge links explicitly. In the following we will neglect the $\mathcal{K}$-terms that are proportional to the momentum transfer to the nucleon. Applying Fierz transformation, restricting ourselves to unpolarized and longitudinal polarized nucleons and taking into account the equations of motion we find

$$
\begin{aligned}
T_{\mu}= & T_{\mu}^{(g)}+T_{\mu}^{(q)}, \\
T_{\mu}^{(g)}= & -\frac{T_{F} q_{f}}{N_{C}} g^{2} \frac{\bar{U}\left(P^{\prime}\right) \phi_{1} U(P)}{2 P q_{1}} \frac{1}{\nu^{2} z_{f}\left(1-z_{f}\right)} \\
& \times \int_{0}^{1} \frac{\mathrm{d} X}{2} \frac{\mathcal{G}_{\zeta}(X)}{(X-\zeta+\mathrm{i} \varepsilon)^{2}(X-\mathrm{i} \varepsilon)^{2}} \\
& \times\left\{\bar{u}\left(p_{f}\right) \not P v\left(p_{f}^{\prime}\right)\left((X-\zeta)^{2}+X^{2}\right) P_{\mu}\right. \\
& \left.+\bar{u}\left(p_{f}\right) \gamma_{\mu} v\left(p_{f}^{\prime}\right)\left((X-\zeta)^{2}+X^{2}-2 x_{p} \zeta^{2}\right) \frac{\nu}{\zeta}\right\} \\
& -\frac{T_{F} q_{f}}{N_{C}} g^{2} \frac{\bar{U}\left(P^{\prime}\right) \gamma_{5} \phi_{1} U(P)}{2 P q_{1}} \frac{1}{\nu^{2} z_{f}\left(1-z_{f}\right)} \\
& \times \int_{0}^{1} \frac{\mathrm{d} X}{2} \frac{\Delta \mathcal{G}_{\zeta}(X)(2 X-\zeta)}{(X-\zeta+\mathrm{i} \varepsilon)^{2}(X-\mathrm{i} \varepsilon)^{2}} \\
& \times\left\{\bar{u}\left(p_{f}\right) \not P \gamma_{5} v\left(p_{f}^{\prime}\right)\left[2 p_{f \mu}-\zeta P_{\mu}\right]+\bar{u}\left(p_{f}\right) \gamma_{\mu} \gamma_{5} v\left(p_{f}^{\prime}\right)\left(1-2 z_{f}\right) \nu\right\},
\end{aligned}
$$




$$
\begin{aligned}
& T_{\mu}^{(q)}=-\frac{1}{4} \frac{C_{F} q_{f}}{N_{C}} g^{2} \frac{\bar{U}\left(P^{\prime}\right) \phi_{1} U(P)}{2 P q_{1}} \frac{1}{\nu^{2} z_{f}\left(1-z_{f}\right)} \\
& \times \int_{0}^{1} \mathrm{~d} X \frac{\left[\mathcal{F}_{\zeta}^{f}(X)+\mathcal{F}_{\zeta}^{\bar{f}}(X)\right](2 X-\zeta)}{(X-\zeta+\mathrm{i} \varepsilon)(X-\mathrm{i} \varepsilon)\left(X-\zeta x_{p}+\mathrm{i} \varepsilon\right)\left(X-\zeta\left(1-x_{p}\right)-\mathrm{i} \varepsilon\right)} \\
& \times\left\{\bar{u}\left(p_{f}\right) \not p v\left(p_{f}^{\prime}\right)\left[\left(X(X-\zeta)+\zeta^{2} x_{p}\left(1-z_{f}\right)\right) P_{\mu}+\zeta x_{p}\left(2 z_{f}-1\right) p_{f \mu}\right]\right. \\
&\left.+\bar{u}\left(p_{f}\right) \gamma_{\mu} v\left(p_{f}^{\prime}\right) \frac{\nu}{\zeta}\left(X(X-\zeta)-\zeta^{2} x_{p}\left(x_{p}-2 z_{f}\left(1-z_{f}\right)\right)\right)\right\} \\
&-\frac{1}{4} \frac{C_{F} q_{f}}{N_{C}} g^{2} \frac{\bar{U}\left(P^{\prime}\right) \phi_{1} U(P)}{2 P q_{1}} \frac{1}{\nu^{2} z_{f}\left(1-z_{f}\right)} \\
& \times \int_{0}^{1} \mathrm{~d} X \frac{\mathcal{F}_{\zeta}^{f}(X)-\mathcal{F}_{\zeta}^{\bar{f}}(X)}{(X-\zeta+\mathrm{i} \varepsilon)(X-\mathrm{i} \varepsilon)\left(X-\zeta x_{p}+\mathrm{i} \varepsilon\right)\left(X-\zeta\left(1-x_{p}\right)-\mathrm{i} \varepsilon\right)} \\
& \times\left\{\overline { u } ( p _ { f } ) \not p _ { v } ( p _ { f } ^ { \prime } ) \left[\left(\zeta X(\zeta-X)\left(1+x_{p}\left(2-4 z_{f}\right)\right)-\zeta^{3} x_{p}\left(1-z_{f}\right)\right) P_{\mu}\right.\right. \\
&\left.+\left(2 X(X-\zeta)+\zeta^{2} x_{p}\right) p_{f \mu}\right] \\
&\left.+\bar{u}\left(p_{f}\right) \gamma_{\mu} v\left(p_{f}^{\prime}\right) \nu\left(X(\zeta-X)-\zeta^{2} x_{p}^{2}\right)\left(2 z_{f}-1\right)\right\} \\
&\left.q_{1} \rightarrow \gamma_{5} \not q_{1}, \not p \rightarrow \not P \gamma_{5}, \gamma_{\mu} \rightarrow \gamma_{\mu} \gamma_{5}, \mathcal{F}^{f} \rightarrow \Delta \mathcal{F}^{f}, \mathcal{F}^{\bar{f}} \rightarrow-\Delta \mathcal{F}^{\bar{f}}\right\} .
\end{aligned}
$$

A typical diagram contributing to $T_{\mu}^{(g)}$ is shown in Fig. 4 on the left, an example for a diagram contributing to $T_{\mu}^{(q)}$ in the same figure on the right. The color factors are denoted in the common way $C_{F}=4 / 3, T_{F}=1 / 2$, and the number of colors $N_{C}=3 . q_{f}$ is the electric charge of the produced quark. It is easy to see that by going to the limit of a massless final state, i.e. $x_{p}=1$, and contracting the amplitude with the polarization vector of a longitudinal polarized photon the results for exclusive meson production are reproduced. See for instance [17]. In such a kinematical limit $z_{f}$ corresponds to the light
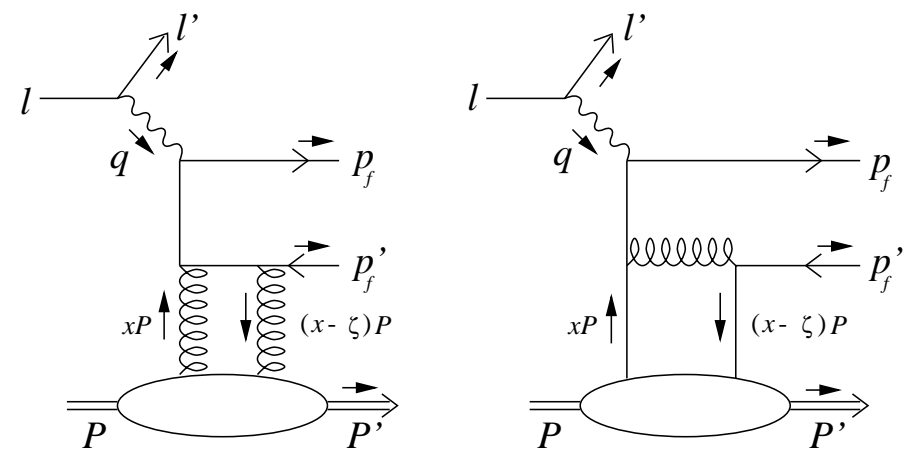

Fig. 4. Typical diagrams to the process involving two gluon emission and two quark emission of the scattered nucleon. 
cone momentum fraction carried by the produced quark. The appearing soft divergences $1 /\left[z_{f}\left(1-z_{f}\right)\right]$ then do not pose any problems for meson wave functions that vanish at the end points. In our case we have to stay off these divergences using cuts for $z_{f}$. Such cuts are demanded by the acceptance of the meson detectors because of the relation $z_{f} \approx z_{\pi}:=P p_{\pi} / P q$. Note that upon contraction with a longitudinal polarization vector and the limit $x_{p}=1$ all dependence on the polarized gluon distribution $\Delta \mathcal{G}$ cancels out.

Contracting $T_{\mu}^{*} T_{\nu}$ with the leptonic tensor $L^{\mu \nu}$ leads us to the differential cross section $\frac{\mathrm{d} \sigma}{\mathrm{d} x_{\mathrm{Bj}} \mathrm{d} y \mathrm{~d} \zeta \mathrm{d} z_{f} \mathrm{~d} t \mathrm{~d} \varphi}$ in terms of the nonforward distributions. To the polarized cross section only the interference term of polarized parton densities and unpolarized densities contribute. All other combinations contribute to unpolarized scattering. For the phenomenological analysis we have to perform the remaining integrations over $X$ in the amplitudes. To do so we need some model for the nonforward parton distributions.

\section{Nonforward Parton Distributions}

The nonforward parton distributions used in the calculations of the previous section are given by an integral over the corresponding double distributions

$$
\mathcal{F}_{\zeta}(X)=\int_{0}^{a} F(X-\zeta y, y) \mathrm{d} y
$$

with $a=\min \left\{\frac{X}{\zeta}, \frac{(1-X)}{(1-\zeta)}\right\}$ [18]. If we write a double distribution as a product of the corresponding usual forward distribution and a profile function

$$
F(x, y)=f(x) \pi(x, y)
$$

the latter has to fulfill the conditions

$$
\pi(x, y)=\pi(x, 1-x-y), \quad \int_{0}^{1-x} \pi(x, y) \mathrm{d} y=1
$$

The symmetry $y \leftrightarrow 1-x-y$ was noted in [17]. As a model for the profile functions we take $\pi(x, y)=\frac{6 y(1-x-y)}{(1-x)^{3}}$ for the quark distributions and $\pi(x, y)=\frac{30 y^{2}(1-x-y)^{2}}{(1-x)^{5}}$ for the gluon distributions [18]. For the (forward) parton distributions we use the (slightly simplified) NLO parameterizations of [19] in the unpolarized and [20] in the polarized case at the scale $Q_{0}^{2}=4 \mathrm{GeV}^{2}$. From 
these models we get the nonforward parton distributions using (13). In the following we will neglect all effects of $Q^{2}$ evolution of the distribution functions. Special care is needed for the quark sea distribution for which the original parton distribution is more singular than $1 / x_{\mathrm{Bj}}$. In this case the integral (13) is not defined for $X \leq \zeta$. The problem can be solved using the anti-symmetry of the remaining part of the corresponding integrand:

$$
\begin{aligned}
\int_{0}^{1} \mathcal{A}(\zeta, X) \mathcal{F}_{\zeta}(X) \mathrm{d} X= & \int_{-1+\zeta}^{\zeta} \mathcal{A}(\zeta, \zeta-X) \mathcal{F}_{\zeta}(\zeta-X) \mathrm{d} X \\
= & \frac{1}{2} \int_{-1+\zeta}^{1} \mathcal{A}(\zeta, X) \\
& \times\left(\mathcal{F}_{\zeta}(X) \Theta(X)-\mathcal{F}_{\zeta}(\zeta-X) \Theta(\zeta-X)\right) \mathrm{d} X \\
= & \int_{\zeta / 2}^{1} \mathcal{A}(\zeta, X) \\
& \times\left(\mathcal{F}_{\zeta}(X)-\mathcal{F}_{\zeta}(\zeta-X) \Theta(\zeta-X)\right) \mathrm{d} X
\end{aligned}
$$

Here the divergences cancel in the difference $\mathcal{F}_{\zeta}(X)-\mathcal{F}_{\zeta}(\zeta-X)$.

In order to determine the cross section integrated over $t$ we assume that the $t$-dependent nonforward distributions factorize into a nucleon form factor and the nonforward distribution at $t=0$

$$
\mathcal{F}_{\zeta}(X, t)=\mathcal{F}_{\zeta}(X, 0) F(t)=\mathcal{F}_{\zeta}(X) F(t)
$$

This behavior is suggested (at least for small values of $t$ ) by the condition $\int_{0}^{1} \mathcal{F}_{\zeta}(X) \mathrm{d} X=F(t)[3]$ (see also [17]). As models for the form factors we take

$$
F(t)=\left(\frac{1}{1-t / \Lambda^{2}}\right)^{\alpha}
$$

with $\alpha=2, \Lambda=0.84 \mathrm{GeV}$ in the case of quark distributions and $\alpha=3$, $\Lambda=2.8 \mathrm{GeV}$ in the case of gluon distributions. The quark form factor is taken from measurements of elastic electron nucleon scattering [21], the gluon form factor from QCD sum rule calculations [22]. Since these form factors decrease rapidly for increasing $|t|$ the integrals over $t$ will be dominated by small $|t|$. Therefore it is justified to keep $t=0$ in the other contributions to the differential cross sections. In this approximation the $t$-integration can be carried out and leads to factors of the form $\int_{-\infty}^{0} F_{1}(t) F_{2}(t) \mathrm{d} t$ where $F_{i}$ are nucleon form factors for quark or gluon fields respectively. 


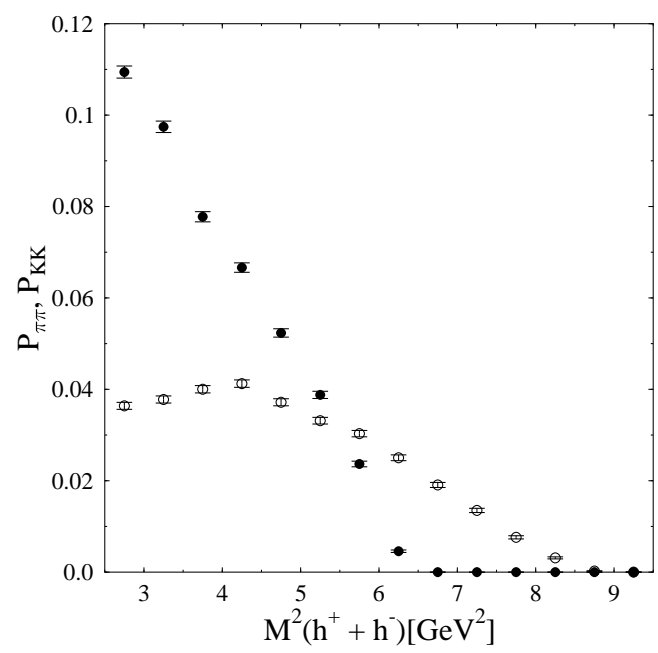

Fig. 5. Probability of creating a $\pi^{+} \pi^{-}$pair (solid circles) or a $K^{+} K^{-}$(open circles) pair in a quark-anti quark fragmentation process with center of mass energy squared $M^{2}\left(h^{+}+h^{-}\right)$.

\section{Fragmentation into Mesons}

To describe the fragmentation process of the two outgoing quarks into a meson pair we use JETSET which is based on the LUND string fragmentation model $[9,10]$. In Fig. 5 we plot the probability to produce a pion or kaon pair from two quarks as a function of the cm-energy $M^{2}\left(h^{+}+h^{-}\right)=\left(p_{f}+p_{f}^{\prime}\right)^{2}=(\zeta P+q)^{2}=$ $2 \nu \zeta\left(1-x_{p}\right)$. For this plot we assumed that all quark-flavors are produced in equal ratios as soon as $M^{2}\left(h^{+}+h^{-}\right)$exceeds the respective mass threshold.

In order to evaluate the cross section for $\pi^{+} \pi^{-}$and $K^{+} K^{-}$pair production in lepton proton scattering the events generated by JETSET are weighted with our differential cross section for quark pair production $\frac{\mathrm{d} \sigma}{\mathrm{d} x_{\mathrm{Bj}} \mathrm{d} y \mathrm{~d} \zeta \mathrm{d} z_{f} \mathrm{~d} \varphi}$. Fig. 5 shows that the center of mass energy squared should be less than $8 \mathrm{GeV}^{2}$ to give sizable meson pair production. In order to avoid regions where we cannot trust the program JETSET any longer we further have to avoid too small values of $M^{2}\left(h^{+}+h^{-}\right)$. For our simulations we work in the proton rest frame with a lepton energy of $27.5 \mathrm{GeV}$ and use an acceptance slightly smaller than the HERMES acceptance. We require the angles of all detected particles (electrons, pions and kaons) with respect to the electron beam axis to lie in the range $0.04<\tan \theta<0.140$. Furthermore, the detected electron should have an energy exceeding $1 \mathrm{GeV}$ and the detected mesons an energy larger than $4 \mathrm{GeV}$. We also require both mesons to have a minimum transverse momentum in the laboratory frame of $0.5 \mathrm{GeV}$. Further we imposed the cut $p_{T}^{2}>1 \mathrm{GeV}^{2}$ (with $p_{T}$ being the transverse momentum in the center of mass 
Table 1

The different contributions to the total cross sections.

\begin{tabular}{ccc}
\hline & cross sect. $\pi^{+} \pi^{-}(\mathrm{pb})$ & cross sect. $K^{+} K^{-}(\mathrm{pb})$ \\
\hline \hline$\sigma_{g g}$ & $3.210^{-1}$ & $2.810^{-1}$ \\
$\sigma_{\Delta g \Delta g}$ & $4.210^{-2}$ & $4.110^{-2}$ \\
$\sigma_{g \Delta g}$ & $6.310^{-2}$ & $5.310^{-2}$ \\
\hline$\sigma_{q q}$ & $5.410^{-2}$ & $4.010^{-2}$ \\
$\sigma_{\Delta q \Delta q}$ & $3.310^{-3}$ & $2.910^{-3}$ \\
$\sigma_{q \Delta q}$ & $7.410^{-3}$ & $4.810^{-3}$ \\
\hline$\sigma_{q g}$ & $-1.210^{-1}$ & $-8.810^{-2}$ \\
$\sigma_{\Delta q \Delta g}$ & $-4.610^{-3}$ & $-7.710^{-4}$ \\
$\sigma_{q \Delta g+g \Delta q}$ & $-4.310^{-2}$ & $-2.910^{-2}$ \\
\hline total spin average & $2.910^{-1}$ & $2.810^{-1}$ \\
total spin asymmetry & $2.810^{-2}$ & $2.910^{-2}$ \\
\hline
\end{tabular}

frame of the photon and the incoming nucleon) in order to keep the relevant scale $\mu^{2}=p_{T}^{2}\left(1+\frac{Q^{2}}{M^{2}\left(h^{+}+h^{-}\right)}\right)$sufficiently large. For the remaining variables we used the cuts $0.02<x_{\mathrm{Bj}}<0.05,0.29<y<0.96,0.1<\zeta<0.3$, and $0.04<$ $z_{f}<0.96$ where the lower cut in $\zeta$ was dictated by numerical reasons. The cuts in $z_{f}$ are part of the $z-s$ jet scheme which we applied in our simulations. It is also used in the standard Monte Carlo leptoproduction programs [23].

\section{Results and Discussion}

In Table 1 we have given the various contributions to the total cross section for meson pair production. Contributions from the polarized and unpolarized gluonic NFPD are denoted by the indices $g$ and $\Delta g$, contributions from the quark NFPDs by $q$ and $\Delta q$ respectively. Since the cross section is quadratic in the parton distributions we find pure gluonic contributions of the form $\sigma_{g g}$, $\sigma_{\Delta g \Delta g}$, and $\sigma_{g \Delta g}$, similar expressions for the pure quarkonic part, and interference contributions $\sigma_{q g}, \sigma_{\Delta q \Delta g}$, and $\sigma_{q \Delta g+g \Delta q}$. Only the interference terms of polarized and unpolarized parton densities $\sigma_{g \Delta g}, \sigma_{g \Delta g}$, and $\sigma_{g \Delta q+q \Delta g}$ depend on the alignment of the spins of the incoming electron and proton and thus contribute to the spin asymmetries $\Delta \sigma:=\frac{1}{2}\left(\sigma_{\uparrow \downarrow}-\sigma_{\uparrow \uparrow}\right)$. ( $\sigma_{\uparrow \downarrow}$ is the cross section for antiparallel lepton spin and nucleon spin.) Comparing the contributions to 
the spin average we see that $\sigma_{\Delta g \Delta g}, \sigma_{\Delta q \Delta q}$, and $\sigma_{\Delta q \Delta g}$ are roughly one order of magnitude smaller than $\sigma_{g g}, \sigma_{q q}$ and $\sigma_{q g}$ respectively.

For our NFPD Models, the gluon contribution dominates the cross section, both in the unpolarized and polarized channel. Note that the normalization of the cross section depends crucially on the form factors (17). For the used input distributions we finally get a spin asymmetry of order $10 \%$.

The unpolarized and polarized cross sections are plotted in the upper pictures in Fig. 6 as functions of the skewedness $\zeta$. The cross sections receive their dominating contribution from the small values of $\zeta$. In our analysis we restrict ourselves on skewedness values larger than $\zeta>0.1$. To go to smaller values of $\zeta$ ultimately would lead to the requirement that $Q^{2} \rightarrow 0$ and thus would lead to the region of photoproduction. In that kinematical domain the scattered electron is not detectable and thus an unambiguous reconstruction of the final hadronic state is not possible unless the outgoing proton could be detected by a recoil spectrometer. One method to distinguish the elastic events from inelastic ones in this case is provided by the existence of a rapidity gap. The plots in the middle of Fig. 6 show that the difference between the minimum of the meson rapidities and the rapidity of the outgoing proton $\Delta y$ is for all diffractive events larger than 2. In the lower diagrams in Fig. 6 we plotted the cross section versus the invariant mass squared of the produced particles. These plots show the increase of the ratio between the kaon and pion rates with the invariant mass $M\left(h^{+}+h^{-}\right)$as suggested by Fig. 5 . The shape of the polarized and unpolarized cross sections is similar.

We have analyzed diffractive $\pi^{+} \pi^{-}$and $K^{+} K^{-}$production as a tool for the determination of polarized and unpolarized nonforward parton distributions. We implemented the realistic cuts for the HERMES experiment and required sufficiently large $p_{T}$ to justify our leading order treatment. With these cuts the cross sections are too small to make this channel a promising one for HERMES. Successfull measurements would probably require an ENC-type machine [24].

\section{Acknowledgements}

The authors thank M. Diehl, P. Hoyer, L. Mankiewicz, G. Piller, M. Polyakov, A. Radyushkin, M. Strikman, and O. Teryaev for useful discussions. This work has been supported by DFG (Graduiertenkolleg), Studienstiftung, and BMBF. M.M. thanks the Nordic Insitute for Theoretical Physics for financial support and hospitality. 

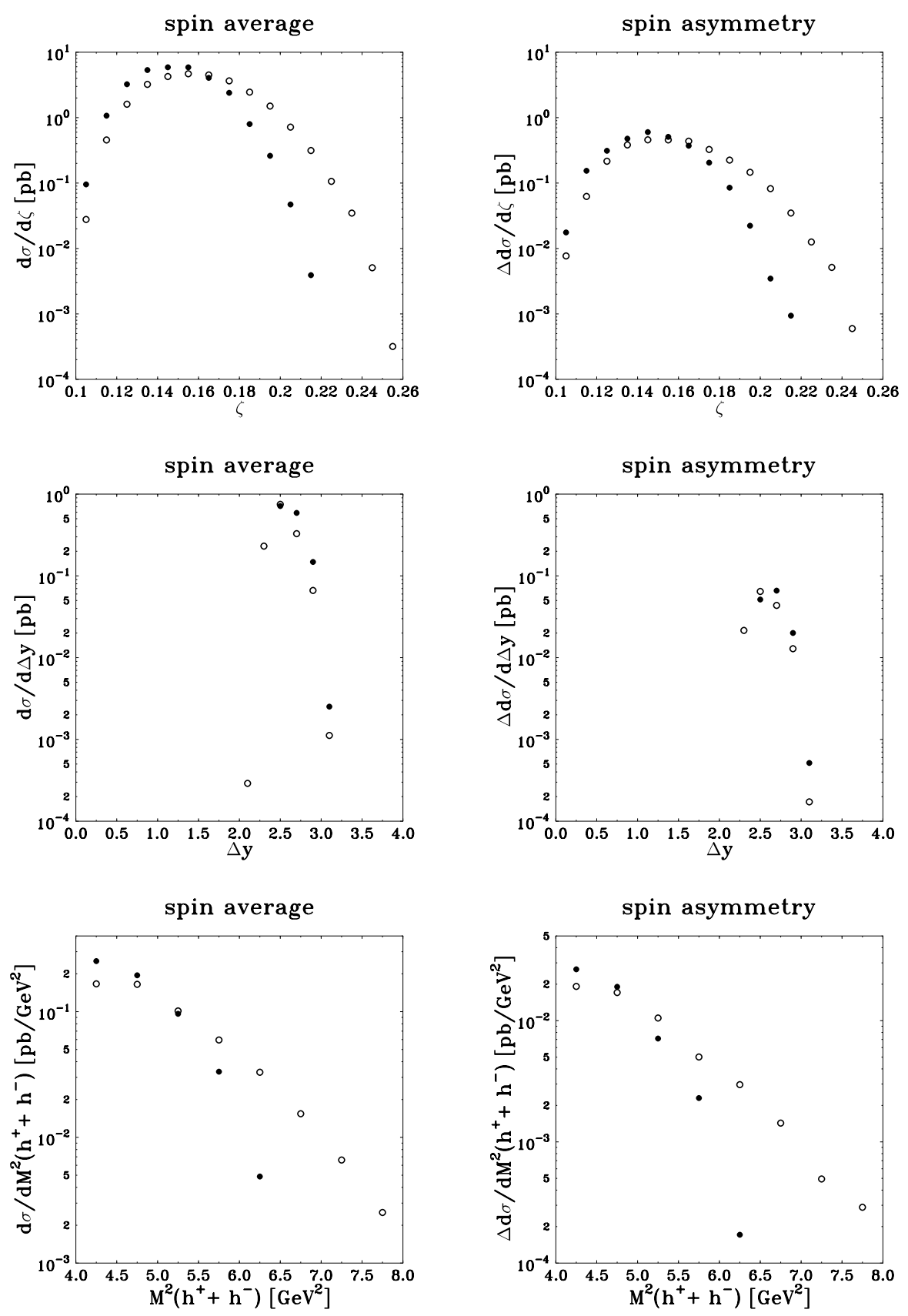

Fig. 6. The unpolarized (on the left) and polarized (on the right) differential cross section for diffractive $\pi^{+} \pi^{-}$(solid circles) and $K^{+} K^{-}$(open circles) production with respect to the variables $\zeta, \Delta y:=\min \left\{y_{\text {particle } 1}, y_{\text {particle } 2}\right\}-y$ outg. proton, and $M^{2}\left(h^{+}+h^{-}\right)$.

\section{References}

[1] J. Bartels, M. Loewe, Z. Phys. C 12, 263 (1982);

B. Geyer, D. Robaschik, M. Bordag, J. Horejsi, Z. Phys. C 26, 591 (1985); 
F.M. Dittes, D. Müller, D. Robaschik, B. Geyer, J. Horejsi, 1988, Phys. Lett. B 209, 325 (1988).

[2] X. Ji, Phys. Rev. D 55, 7114 (1997);

X. Ji, W. Melnitchouk, X. Song, Phys. Rev. D 56, 5511 (1997);

J. Blümlein, B. Geyer, D. Robaschik, Phys. Lett. B 406, 161 (1997);

I.I. Balitsky, A.V. Radyushkin Phys. Lett. B 413, 114 (1997).

[3] A. Radyushkin, Phys. Lett. B 380, 417 (1996); Phys. Lett. B 385, 333 (1996); Phys. Rev. D 56, 5524 (1997).

[4] A.V. Belitsky, D. Müller, Phys. Lett. B 417, 129 (1998);

A.V. Belitsky, B. Geyer, D. Müller, A. Schäfer, Phys. Lett. B 421, 312 (1998);

A.V. Belitsky, D. Müller, L. Niedermeier, A. Schäfer, Phys. Lett. B 437, 160 (1998); hep-ph/9810275.

[5] X. Ji, Phys. Rev. Lett. 78, 610 (1997).

[6] M. Diehl, Z. Phys. C 66, 181 (1995).

[7] K.J. Golec-Biernat, A. D. Martin, Phys. Rev. D 58, 094001 (1998).

[8] A. Bravar, D. von Harrach, A. Kotzinian, Phys. Lett. B 421, 349 (1998).

[9] X. Artru, G. Mennessier Nucl. Phys. B 70, 93 (1974);

B. Andersson, G. Gustafson, G. Ingelman, T. Sjöstrand, Phys. Rep. 97, 31 (1983);

B. Andersson, G. Gustafson, C. Peterson Z. Phys. C 1, 105 (1979);

B. Andersson, G. Gustafson, B. Söderberg Z. Phys. C 20, 317 (1983).

[10] T. Sjöstrand, PYTHIA 5.7 and JETSET 7.4: physics and manual, long version of publication in Comput. Phys. Commun.,

LU-TP-95-20, August 1995,

e-Print Archive: hep-ph/9508391.

[11] J.C. Collins, L. Frankfurt, M. Strikman, Phys. Rev. D 56, 2982 (1997).

[12] S. J. Brodsky, L. Frankfurt, J.F. Gunion, A.H. Mueller, M. Strikman, Phys. Rev. D 50, 3134 (1994).

[13] M. Diehl, T. Gousset, B. Pire, O. Teryaev Phys. Rev. Lett. 81, 1782 (1998).

[14] M.V. Polyakov, Study of two-pion light-cone distribution amplitudes in the resonance region and at low energies, e-Print Archive: hep-ph/9809483.

[15] J. Bartels, H. Lotter, M. Wüsthoff, Phys. Lett. B 379, 239 (1996).

[16] R.D. Peccei, R. Rückl, Nucl. Phys. B 162, 125 (1980).

[17] L. Mankiewicz, G. Piller, T. Weigl, Eur. Phys. J. C 5, 119 (1998).

[18] A.V. Radyushkin, hep-ph/9805342.

[19] A.D. Martin, W.J. Stirling, R.G. Roberts, Phys. Lett. B 354, 155 (1995). 
[20] T. Gehrmann, W.J. Stirling, Phys. Rev. D 53, 6100 (1996).

[21] T. Ericson, W. Weise, Pions and Nuclei, Oxford University Press, 1988.

[22] V.M. Braun, P. Gornicki, L. Mankiewicz, A. Schäfer, Phys. Lett. B 302, 291 (1993).

[23] G. Ingelman, A. Edin, J. Rathsman, Comput. Phys. Commun. 101, 108 (1997).

[24] GSI Report 97-04, Seeheim 1997. 